\title{
Spectroscopy of Collective Excitations in Interacting Low-Dimensional Many-Body Systems Using Quench Dynamics
}

\section{Citation}

Gritsev, Vladimir, Eugene Demler, Mikhail Lukin, and Anatoli Polkovnikov. 2007. “Spectroscopy of Collective Excitations in Interacting Low-Dimensional Many-Body Systems Using Quench

Dynamics." Physical Review Letters 99 (20) (November 16). doi:10.1103/physrevlett.99.200404.

\section{Published Version}

doi:10.1103/PhysRevLett.99.200404

\section{Permanent link}

http://nrs.harvard.edu/urn-3:HUL.InstRepos:27891673

\section{Terms of Use}

This article was downloaded from Harvard University's DASH repository, and is made available under the terms and conditions applicable to Other Posted Material, as set forth at http:// nrs.harvard.edu/urn-3:HUL.InstRepos:dash.current.terms-of-use\#LAA

\section{Share Your Story}

The Harvard community has made this article openly available.

Please share how this access benefits you. Submit a story.

\section{Accessibility}




\title{
Spectroscopy of Collective Excitations in Interacting Low-Dimensional Many-Body Systems Using Quench Dynamics
}

\author{
Vladimir Gritsev, ${ }^{1}$ Eugene Demler, ${ }^{1}$ Mikhail Lukin, ${ }^{1}$ and Anatoli Polkovnikov ${ }^{2}$ \\ ${ }^{1}$ Department of Physics, Harvard University, Cambridge, Massachusetts 02138, USA \\ ${ }^{2}$ Department of Physics, Boston University, Boston, Massachusetts 02215, USA \\ (Received 16 February 2007; revised manuscript received 11 September 2007; published 16 November 2007)
}

\begin{abstract}
We study the problem of rapid change of the interaction parameter (quench) in a many-body lowdimensional system. It is shown that, measuring the correlation functions after the quench, the information about a spectrum of collective excitations in a system can be obtained. This observation is supported by analysis of several integrable models and we argue that it is valid for nonintegrable models as well. Our conclusions are supplemented by performing exact numerical simulations on finite systems. We propose that measuring the power spectrum in a dynamically split 1D Bose-Einsten condensate into two coupled condensates can be used as an experimental test of our predictions.
\end{abstract}

PACS numbers: 03.75.Lm, 03.75.Kk, 03.75.Nt

In many cases interesting quantum phenomena appear not in properties of the ground states but in the coherent dynamics of the system away from equilibrium. Canonical examples from basic quantum mechanics include Rabi oscillations in two level systems, collapse and revival in the Jaynes-Cummings model, and Landau-Zener tunneling. Analysis of nonequilibrium coherent dynamics in a strongly correlated many-body system is a more challenging task than in single-particle quantum mechanics; hence, progress has been made only in a few cases. This includes changing parameters in the transverse field Ising model [1], mean field dynamics in systems with BCS pairing of fermions [2], collapse and revival phenomena [3], and nonequilibrium superfluid phase oscillations [4] of bosonic atoms in optical lattices. In this Letter we discuss another example of nonequilibrium coherent dynamics in strongly correlated many-body systems. We consider a sudden quench of interaction parameter in nonlinear interacting systems and show that the subsequent time dynamics exhibit oscillations with frequencies given by the poles of the scattering matrix of this many-body system. The sudden quench can be thus used as a tool for spectroscopy of the interacting models. We make our analysis by increasing complexity of examples: we start with a quantum Josephson junction (QJJ) model then generalize it for a Gaussian model, and support the main observation on the quantum sine-Gordon model (QSG). At the end we generalize the main statement for nonintegrable models and make supporting arguments in favor of our conclusion for general models.

The QSG model is a popular prototypical example of a nonlinear interacting quantum system. This model appears as an effective description of variety of condensed-matter, statistical physics, and field theory problems. Thus in condensed matter it describes low-dimensional spin and charge systems, disordered systems (see [5] for a review) and coupled Bose condensates [6]. Given the large freedom of tunability of parameters in ultracold quantum gases we have in mind the test of a theory presented below in these dynamically decoupled 1D condensates.

The dynamics in the QSG model can be analyzed using its exact solution. In general, describing dynamical properties of a many-body system using the exact Bethe ansatz solution is not a straightforward procedure. In our case, progress can be made connecting the problem of time evolution from a certain initial state to the equilibrium sine-Gordon model with a boundary. For conformally invariant models a similar approach of mapping temporal evolution after a quench to a class of boundary phenomena was discussed in Ref. [7]. In our case, the system can have different mass scales corresponding to solitons and their bound states.

The Hamiltonian of the QSG model is given by

$$
\mathcal{H}_{\mathrm{SG}}=\frac{1}{2} \int d x\left[\Pi^{2}(x)+\left(\partial_{x} \phi\right)^{2}-4 \Delta \cos (\beta \phi)\right] .
$$

In applications to split condensates the $\phi(x)=\phi_{1}-\phi_{2}$ is the relative phase between the two of them and $\Pi(x)$ is the conjugate momentum proportional to the difference between local densities and $\beta=\sqrt{2 \pi / K}[6]$. The interference experiments, such as reported in Ref. [8], measure the $\phi(x)$ between the two condensates. After the splitting, the system is in a state which is not an eigenstate; hence, this initial state will undergo a complicated quantum dynamics controlled by the many-body Hamiltonian. We assume that at $t=0$ the system is prepared in a state with $\phi=0$ for all $x$. In reality $\phi$ is a wave packet with the width determined either by the rate with which the condensates were separated [9] or by the depletion [10].

The Luttinger parameter $K$ of individual condensates in Eq. (1) is large for weakly interacting bosons, and approaches 1 in the hard core repulsion (Tonks-Girardeau) regime. $K \leq 1$ for fermionic and spin systems. Generally $K$ can be extracted from the microscopics [5].

We concentrate our analysis here on dynamical properties of one-point correlation function, however, general- 
ization of our approach for the multipoint correlation functions is straightforward. The one-point correlation function $\int_{0}^{L} d x\langle\psi(t=0)| \exp (i \beta \phi(x, t)|\psi(t=0)\rangle$ corresponds to the amplitude of interference fringes, $A(t)$ [8] and can be measured as a function of the evolution time $t$. To characterize the time evolution of $A(t)$ we analyze the power spectrum $P(\omega)=\lim _{T \rightarrow \infty}\left|\int_{0}^{T} d t e^{i \omega t} A(t)\right|^{2}$.

It is instructive to analyze first a simpler situation, when the spatial fluctuations of the phase $\phi$ are energetically forbidden and the Hamiltonian (1) reduces to the QJJ model,

$$
\mathcal{H}_{\mathrm{JJ}}=\frac{E_{c}}{2}\left(\frac{\partial}{i \partial \phi}\right)^{2}-J \cos (\phi)
$$

with $E_{c}=\beta^{2} \hbar v_{s} / L$ and $J=2 L \Delta$, where $v_{s}$ is a sound velocity. Following the standard approach for analyzing sudden perturbations in quantum mechanics we decompose the initial state into eigenstates of the Hamiltonian (2), $|\psi(t=0)\rangle=\sum_{n} a_{n}|n\rangle$. Eigenstates $|n\rangle$ can be found explicitly using Matthieu's functions [11]. Only even $n$ 's are present in decomposition of $|\psi(t=0)\rangle$ and the occupation probabilities $\left|a_{n}\right|^{2}$ decrease with increasing $n$, provided that the initial state has a finite width. Letting the system evolve for time $t$, we find a state $|\psi(t)\rangle=$ $\sum_{n} a_{n} e^{-i \omega_{n} t}|n\rangle$. The amplitude $\quad A(t)=\langle\Psi(t)| \times$ $\cos (\phi)|\Psi(t)\rangle=\sum_{n m} a_{n}^{*} a_{m}(\cos \phi)_{n m} e^{-i\left(\omega_{n}-\omega_{m}\right) t}, \quad$ where $(\cos \phi)_{n m}$ is the matrix element of $\cos \phi$ between the states $n$ and $m$. The power spectrum for a specific set of parameters is shown in Fig. 1. The inset on this graph shows the energy level structure and a couple of possible oscillation frequencies.

The power spectrum consists of different peaks corresponding to the overlaps between various even energy levels. The largest (central) peak in the power spectrum comes from beating of $n=0$ and $n=2$ states [i.e., the $(\cos \phi)_{20} e^{i\left(\omega_{2}-\omega_{0}\right) t}+$ c.c. term]. The dominance of this

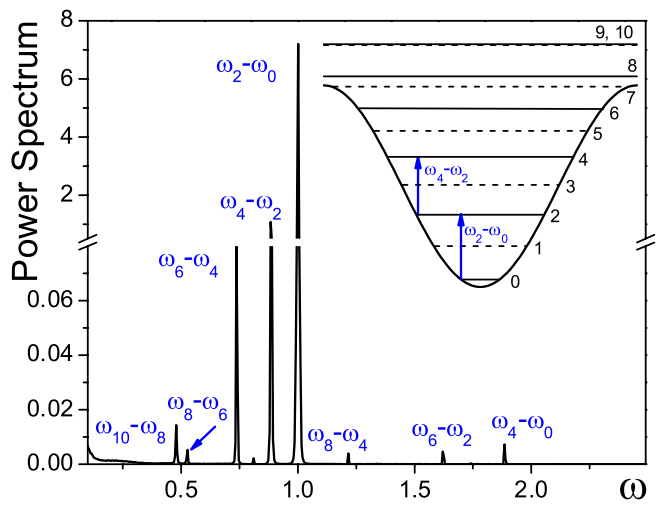

FIG. 1 (color online). Power spectrum for a single QJJ consisting of two sites. At $t=0$ the tunneling amplitude is suddenly reduced from a very large value to $J=10$, the interaction strength $E_{c}=1$. The inset shows the energy levels of the QJJ with the arrows indicating a couple of possible oscillation frequencies. peak comes from a combination of the two factors: (i) The matrix elements $(\cos \phi)_{n m}$ are the largest for the smallest difference between $n$ and $m$ (ii) The state $n=0$ has the largest weight in the decomposition of $|\psi(t=0)\rangle$. There are also contributions at frequencies $\omega_{m}-\omega_{0}$ for $m=4,6, \ldots$ (only $m=4$ is shown), which give rise to peaks at higher frequencies. For a harmonic potential such peaks would appear at precise multiples of the frequency of the central peak. But because of the unharmonicity such peaks are shifted to lower frequencies. Similarly, other important contributions to the power spectrum come from beating between states $n$ and $n+2$ with $n>0$. They appear at smaller frequencies than that of the central peak. Finally, we have contributions from beatings of various other combinations of states, but they come with a smaller weight.

We now discuss a case of the full 1D model (1). In the large $K$ limit, the QSG model can be well approximated by the Gaussian theory with a massive term $\sim m \phi^{2}$, where $m \sim J_{\perp}$, instead of the cosine. The initial state is then a product of squeezed states for all $k$ vectors $\left|\psi_{0}\right\rangle \sim$ $\prod_{k} \exp \left(a_{k}^{\dagger} a_{-k}^{\dagger}\right)|0\rangle$, where $a_{k}^{\dagger}$ is the usual bosonic creation operator for the excitation with the momentum $k$ and the energy $\omega_{k}=\sqrt{k^{2}+m^{2}}$. Expanding it, one observes that the state $\left|\psi_{0}\right\rangle$ contains various combinations of pairs of particles with opposite momenta. The leading contribution to $A(t)$ coming from two-particle excitations behaves at long times as $\langle\cos [\phi(x, t)]\rangle \sim \sin (2 m t) / \sqrt{t}$. This behavior leads to the power law singularity in the power spectrum: $P(\omega) \sim|\omega-2 m|^{-1}$. Higher harmonics corresponding to multiparticle excitations correspond to weaker singularities and their weights are suppressed.

With decreasing $K$, nonlinearities start to play an increasingly important role. As we will show below, the peak at fundamental frequency $\omega_{0}$ splits into a sharp singularity and a two-particle contribution (see Fig. 2). The twoparticle contribution corresponds to the excitation of a pair of two lowest energy breathers $B_{1}$, which are direct analogues of the massive excitations in the Gaussian model. The singularity corresponds to the nondecaying excitation of an isolated $B_{2}$ breather, which is a bound state of two $B_{1}$ breathers (for more explicit notations see below). In addition to splitting of the fundamental peak one finds that higher harmonics are shifted to lower frequencies in a direct analogy with a QJJ. Beatings of different harmonics lead to the appearance of singular peaks at frequencies smaller than $\omega_{0}$, similarly to a QJJ system.

For finite $K$ a convenient complete basis of the QSG model is provided by the asymptotic scattering states which can be obtained by the action of the elements $A_{a_{k}}(\theta)$ of the Zamolodchikov-Faddeev algebra [12] on the vacuum state: $\left|\theta_{1} \theta_{2} \ldots \theta_{n}\right\rangle_{a_{1}, a_{2}, \ldots, a_{n}}=A_{a_{1}}^{\dagger}\left(\theta_{1}\right) \times$ $A_{a_{2}}^{\dagger}\left(\theta_{2}\right) \ldots A_{a_{n}}^{\dagger}\left(\theta_{n}\right)|0\rangle$. Here the operators $A_{a_{k}}\left(\theta_{k}\right)$ have internal index $a_{k}$ (corresponding to solitons (+), antisolitons ( - ), or breathers $(n)$ [denoted by $B_{n}$ below)] and depend on the rapidity variable $\theta_{k}$, defining the momentum 


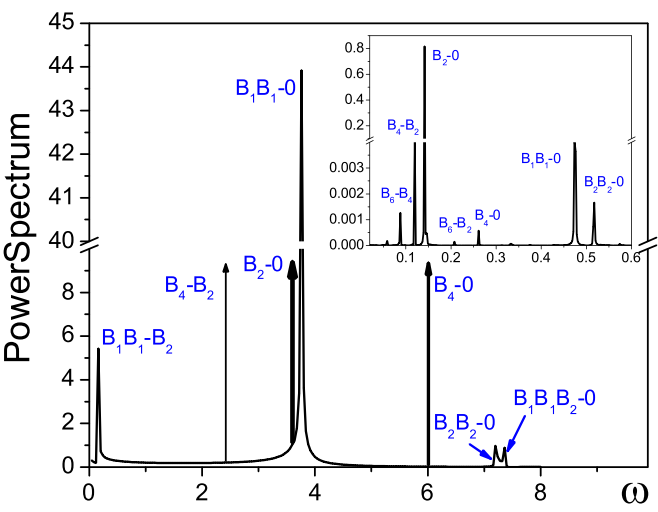

FIG. 2 (color online). Power spectrum for $K=1.6$ and $\Delta=$ 0.4 including single and two-breather contributions. Many other contributions are not visible on this scale. Arrows indicate $\delta$-peaks of type (i) (see the text below). The inset shows the power spectrum from the exact diagonalization on $6 \times 2$ sites for the bosonic Hubbard model. Note the break in the vertical scale. Frequency units are defined by $M_{s}(\Delta, K)$ for given $\Delta$ and $K$.

and the energy of a single quasiparticle excitation of mass $M_{a}: E=M_{a} \cosh (\theta), P=M_{a} \sinh (\theta)$. The operators are defined in such a way that $A_{a_{k}}\left(\theta_{k}\right)|0\rangle=0, \forall k, a_{k}$. Explicit dependence of $M_{a}(\Delta)$ was found in Ref. [13].

Next we consider the initial condition $\phi(t=0)=0$ as a boundary-in-time condition of the Dirichlet type. It belongs to the class of integrable initial (boundary) conditions, which generically have the following form [14]:

$$
|B\rangle=\mathcal{N} e^{\sum_{a, b, n}\left(\left(g_{2 n} / 2\right) B_{2 n}^{\dagger}(0)+\int_{-\infty}^{\infty}(d \theta / 4 \pi) K^{a b}(\theta) A_{a}^{\dagger}(-\theta) A_{b}^{\dagger}(\theta)\right)}|0\rangle .
$$

The matrix $K$ is related to the reflection matrix of solitons, antisolitons, and breathers. These matrices are known [14] for all integrable boundary conditions. The important feature of these boundary states is the presence of various poles in the reflection amplitudes $K^{a b}$. These poles correspond to bound states of the particles $A_{a}, A_{b}$. In the solitonantisoliton channel such bound states are breathers with masses $M_{B_{n}}=2 M_{s} \sin (n \pi /(8 K-2))$, where $n=$ $1, \ldots, 4 K-1$ and $M_{s} \equiv M_{s}(\Delta, K)[13]$.

The poles in the soliton-antisoliton reflection amplitude $\mathrm{K}^{+-}$imply the possibility of exchanging breathers with zero momentum, $B_{a}(0)$. The corresponding " $+-B_{a}$ " coupling is denoted by $g_{a}$. For more discussions and results on the structure of boundary states see [15]. We note that the state (3) is a generalization of squeezed states in the Gaussian theory. The presence of additional breather terms at zero rapidity is the consequence of unharmonicity of the underlying model. Another important difference with the harmonic theory are the unusual commutation relations between $A_{a}$ 's which are neither bosons nor fermions. The state (3) is the initial state in our problem. The Hamiltonian (1) is diagonal in $A$ 's, which allows us to compute the time dependence of $\left|\psi_{B}\right\rangle$

$$
\begin{aligned}
\left|\psi(t)_{B}\right\rangle= & \mathcal{N} \exp \left\{\sum_{a, b, n} \frac{g_{2 n}}{2} B_{2 n}^{\dagger}(0) e^{-i M_{B_{2 n}} t}+\int_{-\infty}^{\infty} \frac{d \theta}{4 \pi}\right. \\
& \left.\times \exp [-i M t \cosh (\theta)] K^{a b}(\theta) A_{a}^{\dagger}(-\theta) A_{b}^{\dagger}(\theta)\right\}|0\rangle,
\end{aligned}
$$

where $M=M_{a}+M_{b}$. For a translation invariant system $A(t)=L\left\langle\psi(t)\left|e^{i \beta \phi(x=0)}\right| \psi(t)\right\rangle$, where $L$ is a system size. We evaluate $A(t)$ by expanding the exponential form of $\left|\psi(t)_{B}\right\rangle$ and computing terms one by one. We use the formfactors (FF) technique discussed by us in Ref. [6] for a related problem (for a general review of the FF approach see Ref. [12]). Our calculations rely on recent progress in evaluation of FF for the QSG model [16]. In general, FF are defined as expectation values of the operators in the asymptotic states $\left|\theta_{1} \ldots \theta_{n}\right\rangle$, which can always be represented in the canonical form, $F^{\mathcal{O}}=\left\langle 0|\mathcal{O}| \theta_{1} \ldots \theta_{n}\right\rangle_{a_{1} \ldots a_{n}}$. It is known from general principles [12] that the FF expansion converges rapidly with increasing number of the participating states. We will therefore discuss the most important contributions to the power spectrum, which come from the first terms in the expansion of Eq. (3). These contributions split into the following categories: (i) Delta function peaks corresponding to contributions of individual breathers. They appear at frequencies $\omega^{(1)}=M_{B_{2 m}}$ and $\omega^{(11)}=$ $M_{B_{2 m}}-M_{B_{2 n}}, m \geq n$. The strengths of these peaks are given by $g_{m} F_{B_{2 m}} / 2$ and $g_{m} g_{n} F_{B_{2 m}} F_{B_{2 n}} / 4$ correspondingly. Here the single-particle breather FF's are [6]:

$$
F_{B_{n}}^{\exp (i \beta \phi)}=\frac{G_{\beta} \sqrt{2} \sin (\pi n \xi) \exp \left[I\left(-\theta_{n}\right)\right] e^{i \pi n / 2}}{\tan \left(\frac{\pi \xi}{2}\right)\left[\cot \left(\frac{\pi \xi n}{2}\right) \prod_{s=1}^{n-1} \cot ^{2}\left(\frac{\pi \xi s}{2}\right)\right]^{1 / 2}},
$$

where $\theta_{n}=i \pi(1-n \xi), \xi=1 /(4 K-1), I(\theta)$ is given in [6] and the numerical factor $G_{\beta}$ was computed in Ref. [17]. These contributions generally decrease with $n$ and decrease with $K$. (ii) Two-particle $A_{a} A_{b}-0$ contributions of excitations with equal masses corresponding to $\omega^{(2)}=$ $2 M_{A_{a}}$. They come from a continuum part. Their weights can be estimated by multiplying corresponding singleparticle FF's. (iii) Interference contributions involving more than two particles of equal or unequal masses.

In Fig. 2 we present the results of our calculations for specific values of $K$ and $\Delta$. For large $K$ the dominant contribution comes from beating of the vacuum state and the $B_{1}(\theta) B_{1}(-\theta)$ pairs corresponding to the massive phonons. The corresponding contribution is absent for a QJJ. The reason is that $B_{1}$ breathers can be excited only with nonzero momentum. However, the nondecaying peaks, corresponding to particles excited at strictly zero momentum, have their direct analogues (see Fig. 1). Indeed the $B_{2}-0$ peak corresponds to the "central peak" in the QJJ picture. The satellite peaks $B_{4}-B_{2}, B_{6}-B_{4}, B_{4}-0$, etc., also have their analogues in Fig. 1. Such peaks correspond to the nondecaying oscillations. Their $\delta$-peak nature is a consequence of the QSG integrability. 
Our analytic results are supported by exact numerical simulations in a system of $N=12$ particles in two chains each of 6 sites. We used the Hubbard model with periodic boundary conditions and with the interaction $U=1$ and intrachain hopping $J=1$ to realize a system with moderate value of $K$ far from the Mott state. At time $t=0$ the interchain hopping $J_{\perp}$ was abruptly decreased from a very large value to $J_{\perp}=0.1$. Despite seemingly small size we point out that this system contains more than $10^{6}$ states, which is more than enough to distinguish integrable versus nonintegrable dynamics (see, e.g., Ref. [18]). The results of such simulations are in complete qualitative agreement with the analytic predictions (see the inset in Fig. 2). We can identify peaks corresponding to various breathers. There are also two additional two-particle contributions denoted as $B_{1} B_{1}-0$ and $B_{2} B_{2}-0$ in the inset. These peaks correspond to excitations with nonzero relative momentum. However unlike in the true thermodynamic limit, these oscillations are not broadened, since there is no continuum of different momentum states.

Previous analysis for the integrable models can be generalized further for nonintegrable models using the formfactor perturbation theory [19]. If we consider not-sostrong deviations from integrability, which means that there are still no multiple particle production in a theory (which is equivalent to the absence of a branch-cuts in a physical strip of a Mandelstam variable), one can argue that the first effect is the change of the position of the poles in the scattering matrix. This variation of pole can be shown to be given by the particle-antiparticle FF $F\left(\theta_{1}-\right.$ $\left.\theta_{2}\right)$, and the change of the particles mass is given by [19] $\delta m_{a}^{2} \sim F_{a \bar{a}}^{\mathcal{O}}(i \pi)$ corresponding to perturbing operator $\mathcal{O}$. Therefore the leading effect of deviation from integrability is a shift of positions of peaks.

It is possible to argue in general that the time evolution of expectation values of some local observable operator in an initial state formed by a sudden quench will provide the information about the spectrum of the theory after this transition. One can indeed show [20] that the boundary reflection amplitude $R(k)$ is related to the bulk Green function $G\left(x, x^{\prime} ; t-t^{\prime}\right)$ as $G\left(x, x^{\prime} ; t-t^{\prime}\right)=\int d \omega \frac{e^{-i \omega\left(t-t^{\prime}\right)}}{4 \pi k(\omega)} \times$ $\left(e^{i k(\omega)\left|x-x^{\prime}\right|}+R(k) e^{i k(\omega)\left|x-x^{\prime}\right|}\right)$ for the field theory with dispersion $k(\omega)$. This formula is somehow reminiscent of the $T$-matrix formulation of the impurity scattering problem. We therefore observe that the poles structure of the Green function is encoded in a boundary reflection factor. Another part of the boundary state is the form factor. The form factors' expectation values can be expanded using the LSZ formula [21], which relates the $n$-particle form factors of operator $\mathcal{O}$ to a $n$-point function. All that leads to the conclusion that spectrum generation during the quench dynamics is a generic phenomena.

In this Letter we show that the dynamics after quench in many-body interacting systems can be used for spectroscopy of collective excitations. We have explicitly demonstrated this on quantum sine-Gordon type models and argued that this conclusion is valid for more general, nonintegrable models. We support our statement for general many-body systems using the connection of the boundary reflection amplitude to the Green's functions.

We would like to acknowledge E. Altman, I. Bloch, R. Cherng, C. Kollath, S. Lukyanov, M. Oberthaler, J. Schmiedmayer, G. Takacs, V. Vuletic for useful discussions. This work was partially supported by the NSF, Harvard-MIT CUA and AFOSR. V. G. is also supported by the Swiss NSF, A. P. is supported by AFOSR YIP.

[1] K. Sengupta, S. Powell, and S. Sachdev, Phys. Rev. A 69, 053616 (2004); R. W. Cherng and L. S. Levitov, Phys. Rev. A 73, 043614 (2006).

[2] E. A. Yuzbashyan, O. Tsyplyatyev, and B. L. Altshuler, Phys. Rev. Lett. 96, 097005 (2006).

[3] M. Greiner, O. Mandel, T. W. Hänsch, and I. Bloch, Nature (London) 419, 51 (2002).

[4] A. K. Tuchman, C. Orzel, A. Polkovnikov, and M. A. Kasevich, Phys. Rev. A 74, 051601 (2006).

[5] T. Giamarchi, Quantum Physics in One Dimension (Oxford University, New York, 2004).

[6] V. Gritsev, A. Polkovnikov, and E. Demler, Phys. Rev. B 75, 174511 (2007).

[7] P. Calabrese and J. Cardy, Phys. Rev. Lett. 96, 136801 (2006).

[8] S. Hofferberth et al., Nature Phys. 2, 710 (2006); G.-B. Jo et al., Phys. Rev. Lett. 98, 030407 (2007).

[9] A. Burkov, M. D. Lukin, and E. Demler, Phys. Rev. Lett. 98, 200404 (2007).

[10] R. Bistritzer and E. Altman, Proc. Natl. Acad. Sci. U.S.A. 104, 9955 (2007); M. A. Cazalilla, Phys. Rev. Lett. 97, 156403 (2006).

[11] I. S. Gradshteyn and I. M. Ryzhik, Table of Integrals, Series, and Products (Academic, New York, 2000).

[12] F. A. Smirnov, Form Factors in Completely Integrable Models of Quantum Field Theory (World Scientific, Singapore, 1992); F.H.L. Essler and R. M. Konik, arXiv:cond-mat/0412421.

[13] Al. B. Zamolodchikov, Int. J. Mod. Phys. A 10, 1125 (1995).

[14] S. Ghoshal and A. Zamolodchikov, Int. J. Mod. Phys. A 9, 3841 (1994); S. Ghoshal, ibid. 9, 4801 (1994).

[15] P. Dorey et al., Nucl. Phys. B594, 625 (2001); Z. Bajnok, G. Bohm, and G. Takacs, ibid. B622, 548 (2002); B682, 585 (2004); Z. Bajnok, L. Palla, and G. Takacs, Nucl. Phys. B772, 290 (2007).

[16] S. Lukyanov, Commun. Math. Phys. 167, 183 (1995); S. Lukyanov, Mod. Phys. Lett. A 12, 2543 (1997).

[17] S. Lukyanov and A. Zamolodchikov, Nucl. Phys. B493, 571 (1997).

[18] S. Mukerjee, V. Oganesyan, and D. Huse, Phys. Rev. B 73, 035113 (2006).

[19] G. Delfino, G. Mussardo, and P. Simonetti, Nucl. Phys. 473, 469 (1996).

[20] Z. Bajnok, G. Bohm, and G. Takacs, J. Phys. A 35, 9333 (2002).

[21] H. Lehmann, K. Symanzik, and W. Zimmermann, Nuovo Cimento 1, 205 (1955). 